\title{
UEVALUA: El uso de las nuevas tecnologías para la evaluación y seguimiento de los estudiantes de Grado en Fisioterapia
}

\author{
Martínez-Pascual, Beatriz ${ }^{1}$, De la Cueva-Reguera , Mónica ${ }^{2}$, García-Mateos, Mónica ${ }^{3}$, \\ Fernández- Martínez, Silvia ${ }^{4}$, González- de-Ramos, Cristina ${ }^{5}$, Blanco- Morales, María ${ }^{6}$. \\ 1,2,3,4,5,6 Facultad de Ciencias Biomédicas y de la Salud, Universidad Europea de Madrid. \\ 1beatriz.martinez@universidadeuropea.es, ${ }^{2}$ monicadelacueva@universidadeuropea.es, \\ ${ }^{3}$ monica.garcia@universidadeuropea.es, ${ }^{4}$ silvia.fernandez@universidadeuropea.es, \\ 다istina.gonzalez@universidadeuropea.es, ${ }^{6}$ maria.blanco@universidadeuropea.es.
}

\begin{abstract}
Universidad Europea de Madrid has implemented in September 2016 a digital platform to facilitate the management, monitoring and assessment of the clinical education taking place in Hospitals and other clinical sites:UEVALUA.

The aim of this qualitative phenomenological study was to describe the experience with this new digital platform among physical therapy students, academic tutors and clinical supervisors making use of the platform in the first quarter of 2016/2017 academic course.

Through a theoretical sampling 13 participants were included. Data were collected using focus groups. A qualitative analysis was conducted using Atlas -Ti v6.0. The identified themes included: a) Difficulties in using the platform; b) The early training; c) Advantages of the platform; d) The impact of the platform on the students'performance and assessment; and d) Improvement proposals.

Keywords: Physical therapy, clinical education, digital learning, assessment, qualitative research, Phenomenology.
\end{abstract}

\section{Resumen}

En el curso 2016/2017 el Grado de Fisioterapia de la Universidad Europea de Madrid ha puesto en marcha una plataforma digital para facilitar la gestión, el seguimiento y la evaluación de las asignaturas de prácticas clínicas que se realizan en hospitales o clínicas: UEVALUA.

El objetivo de este estudio es conocer la experiencia vivida respecto a esta nueva plataforma por parte de los estudiantes y tutores académicos y clínicos del Grado en Fisioterapia, que han utilizado la nueva plataforma en el primer trimestre del curso 2016/2017.

Se ha planteado un diseño cualitativo de tipo fenomenológico descriptivo. Se ha utilizado un muestreo teórico con un tamaño final de la muestra de 13 individuos. La herramienta de recogida de datos utilizada ha sido el Grupo de Discusión. Los datos se han analizado con software Atlas-Ti v6.0. Los temas identificados incluyen : a) Complicaciones con el manejo de la plataforma; b) La formación inicial; c) Ventajas de la plataforma; d) El 
impacto del uso de una herramienta digital sobre el rendimiento y evaluación de los estudiantes, y d) Propuestas de mejora.

Palabras clave: Fisioterapia, formación clínica, aprendizaje digital, evaluación, investigación cualitativa, fenomenología.

\section{Introducción}

La formación de Grado en Fisioterapia está regulada por la ORDEN CIN/2135/2008, de 3 de julio, donde se establecen los objetivos y competencias que todo estudiante debe adquirir para el desarrollo de la profesión.

Las prácticas académicas externas constituyen una actividad de naturaleza formativa realizada por los estudiantes universitarios y supervisada por la Universidad. Su objetivo es permitir a los mismos aplicar y complementar los conocimientos adquiridos en su formación académica, favoreciendo la adquisición de competencias que les preparen para el ejercicio de actividades profesionales, faciliten su empleabilidad y fomenten su capacidad de emprendimiento según el Real Decreto 592/2014, de 11 de julio por el que se regulan las prácticas académicas externas de los estudiantes universitarios.

El modelo académico de la Universidad Europea apuesta por una enseñanza basada en competencias que desarrolla gracias a la realización de las diferentes asignaturas de prácticas externas (Estancias clínicas I y II, Practicum) que se imparten en el Grado en Fisioterapia, formación que da acceso a una profesión regulada por la Ley 44/2003, de 21 de noviembre, de Ordenación de las Profesiones Sanitarias.

El objetivo fundamental de la Plataforma Digital de Prácticas Clínicas (UEVALUA) es facilitar la gestión de estas actividades formativas, así como el seguimiento y la evaluación de los objetivos de aprendizaje asociados con cada una de las asignaturas, resolviendo de esta forma las dificultades del anterior sistema.

Esta plataforma facilita el nexo entre el tutor clínico, el tutor académico de la Universidad, el Coordinador Académico de Prácticas y el estudiante. Es además un soporte informático donde quedan registradas todas las actividades formativas del estudiante, desde los documentos propios, a las calificaciones, evitando así la disparidad de documentos y lo inadecuado de los mismos (Martinez y Estrany et al., 2014).

Cabe destacar que las plataformas e-learning nos permiten crear nuevos espacios de aprendizaje en internet, con una amplia gama de funcionalidades, que sin duda puede resultar difícil de entender, usar y mantener, por el personal docente no informático, por

(cc)) BY-NC-ND 2017, Universitat Politècnica de València 
ello, sólo es posible utilizar las e-learning si se dispone de campus virtuales, y dicho uso mejora el trabajo académico, la calidad de la enseñanza aprendida y la optimización de recursos (Fernández-Pampillon, 2009).

No hay que olvidar que contribuye a un Campus más Ecológico, puesto que el uso del papel se ve disminuido considerablemente ayudando al cuidado del medioambiente. La implementación de esta nueva ayuda tecnológica es sin duda un avance en la gestión y sobretodo en el seguimiento de la formación de los estudiantes de grado.

\section{Objetivos}

\subsection{Objetivo general}

1. Conocer la experiencia que aporta la plataforma a los estudiantes.

2. Conocer la experiencia que aporta la plataforma al tutor clínico y al tutor académico de prácticas de la universidad.

\subsection{Objetivos específicos}

1. Observar el impacto de la herramienta en la comunicación entre estudiantes y tutores.

2. Analizar el impacto de la herramienta en el proceso de evaluación de las prácticas clínicas en los estudiantes.

3. Aportar propuestas de mejora para su desarrollo en el próximo curso académico.

\section{Desarrollo de la innovación}

Para dar respuesta a nuestros objetivos de investigación es necesario el estudio de las experiencias y la construcción de significados lo que nos dirige a una metodología cualitativa (Starks y Trinidad, 2007). Klopper (2008) señala que la investigación cualitativa aporta datos y participa en la generación de hipótesis para posteriores trabajos en diferentes contextos como la clínica, la gestión, la docencia y la investigación.

En la presente investigación se analiza el impacto de la plataforma UEVALUA vista desde diferentes enfoques y por tanto aporta una visión más real del impacto de la nueva plataforma en los participantes.

La importancia de la metodología cualitativa radica en que aporta datos para ayudar a comprender el qué, el cómo y por qué (Sheldon, 2005). Esto comprende describir los mecanismos detrás de los procesos y acciones de los profesionales, comprender resultados heterogéneos, identificar factores que pueden influir en la implementación de una intervención, describir las experiencias de las personas que reciben esa intervención, y describir los resultados desde el punto de vista de los participantes (Centre for Reviews and Dissemination, 2008). 
Dentro de la metodología cualitativa, este estudio se enmarca dentro de un diseño de tipo fenomenológico descriptivo por grupos de discusión (Giorgi 2012; 2005). En él se describirá la experiencia vivida durante la evaluación y seguimiento de las prácticas clínicas de estudiantes y tutores del grado en fisioterapia a través de la plataforma UEVALUA.

\subsection{Población a estudio}

Para la participación en este estudio se realizó una muestra de conveniencia pues en este proyecto no se pretendía generalizar sino profundizar de manera concreta en los individuos y su situación (Pérez-Serrano, 1994). El muestreo intencional de tipo teórico no obedece a unas reglas fijas, ni especifica de antemano el número de unidades a seleccionar. Se orienta a la selección de aquellas unidades y dimensiones que le garanticen mejor la cantidad (saturación) y la calidad y riqueza de la información.

\subsection{Criterios de inclusión}

- Mayores de 18 años.

- Firma del consentimiento informado.

Tabla 1. Selección de participantes.

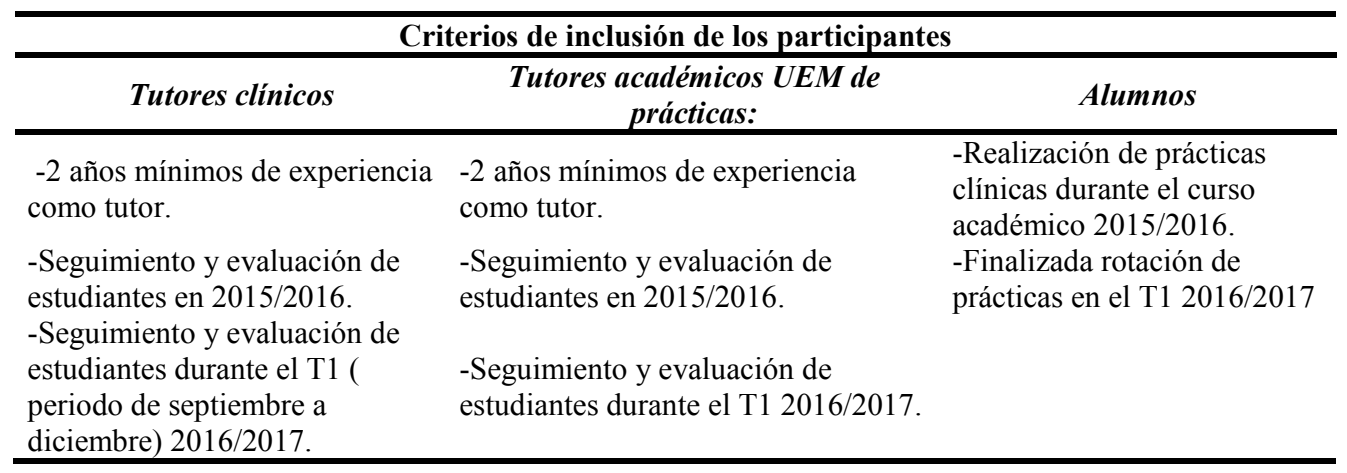

\subsection{Criterios de exclusión}

- Sujetos que no firmen el consentimiento informado.

- Aquellas personas que no consientan realizar grabación de audio.

\subsection{Muestreo por conveniencia}

- Centros u hospitales que cumplan los criterios para los tutores clínicos.

- Profesores del departamento de fisioterapia que habían tutorizado alumnos en prácticas y que cumplían los criterios de inclusión y exclusión.

- Alumnos que habían finalizado la rotación en T1, comprobando aquellos que cumplían los criterios de inclusión. 
Los datos obtenidos permitieron realizar una primera toma de contacto vía correo electrónico, informando de manera individual a los sujetos identificados del proyecto que íbamos a llevar a cabo y preguntando por su disponibilidad para realizar un grupo de discusión.

\subsection{Técnicas de recogida de la información}

Con las respuestas obtenidas se realizan 3 grupos de discusión; uno de alumnos, otro de tutores académicos y por último, uno de tutores clínicos.

\section{Grupos de discusión}

El moderador del grupo de discusión trata de permitir una discusión libre y al mismo tiempo moderar hacia una particular dirección de acuerdo con los temas que conviene tratar.

Para ello el moderador tiene un guion (Blández, 2000). Se inicia el grupo de discusión con una presentación en la que se informa de las normas del grupo de discusión y a continuación se plantean unas preguntas clave.

\section{Recogida de datos}

Los grupos de discusión fueron grabados en audio, durante las sesiones programadas.

Pérez Serrano (1994) los encuadra en los llamados elementos de registro mecánico. La grabación en audio permite registrar con exactitud una conversación o debate oral.

Las grabaciones en audio permiten volver a escuchar lo que se ha dicho, tanto en los escenarios de la acción, como en los grupos de discusión. Para ello se utilizó una grabadora Olympus modelo VN-731PC. Se informaba al participante de la grabación, tanto de manera escrita a través del consentimiento informado como de manera oral previa al comienzo de cada entrevista.

\subsection{Procedimiento de procesamiento, tratamiento y análisis de datos}

El análisis de los resultados se realizó mediante el programa informático de análisis cualitativo Atlas-Ti v6.0. Este es un programa de análisis cualitativo de carácter inductivo. El objetivo de este programa es facilitar el análisis cualitativo de grandes volúmenes textuales, archivos de sonido, imagen o vídeo. Es de gran ayuda a la hora de agilizar el análisis cualitativo y su interpretación como, por ejemplo, la segmentación de textos en pasajes o citas, la codificación o la escritura de comentarios y anotaciones (Muñoz, 2008). Para asegurar los criterios de credibilidad y rigor (Lincoln y Guba, 1985) se realiza una triangulación de los participantes ya que se recoge información de tres colectivos diferentes, los alumnos, los tutores clínicos y los tutores académicos, hecho que asegura la validez interna del estudio. La validez externa se asegurar con la descripción exhaustiva que se realiza en el apartado de introducción sobre la asignatura de estancias clínicas II y el 
sistema educativo universitario Español, contexto donde se desarrolla la presente investigación.

\section{Resultados}

Este epígrafe se compone de 5 apartados en relación a cada una de las redes obtenidas tras el análisis de los datos recogidos a través de las grabaciones en audio de los grupos de discusión: Complicaciones del uso de la nueva plataforma respecto al manejo, Formación inicial sobre el manejo de la plataforma, Impacto de la plataforma en el rendimiento del alumno, Ventajas del uso de la plataforma y Propuestas de mejora.

Las citas se nombran según el instrumento de recogida de la información (GD para grupos de discusión), la persona que emitió dicha información (A para alumnos, TC para los tutores clínicos y TA para el tutor académico) y el minuto del audio donde se recoge la información.

\subsection{Complicaciones del uso de la nueva plataforma respecto al manejo}

Esta familia está compuesta por un total de 30 códigos. Se divide en tres subcategorías o subfamilias: Aplicación móvil de la plataforma, Falta de claridad en el User Experience de la plataforma y los Problemas de accesibilidad. La figura que se muestra a continuación representa el árbol-estructura sobre las dificultades encontradas por los participantes durante la utilización de la plataforma UEVALUA (Fig1).

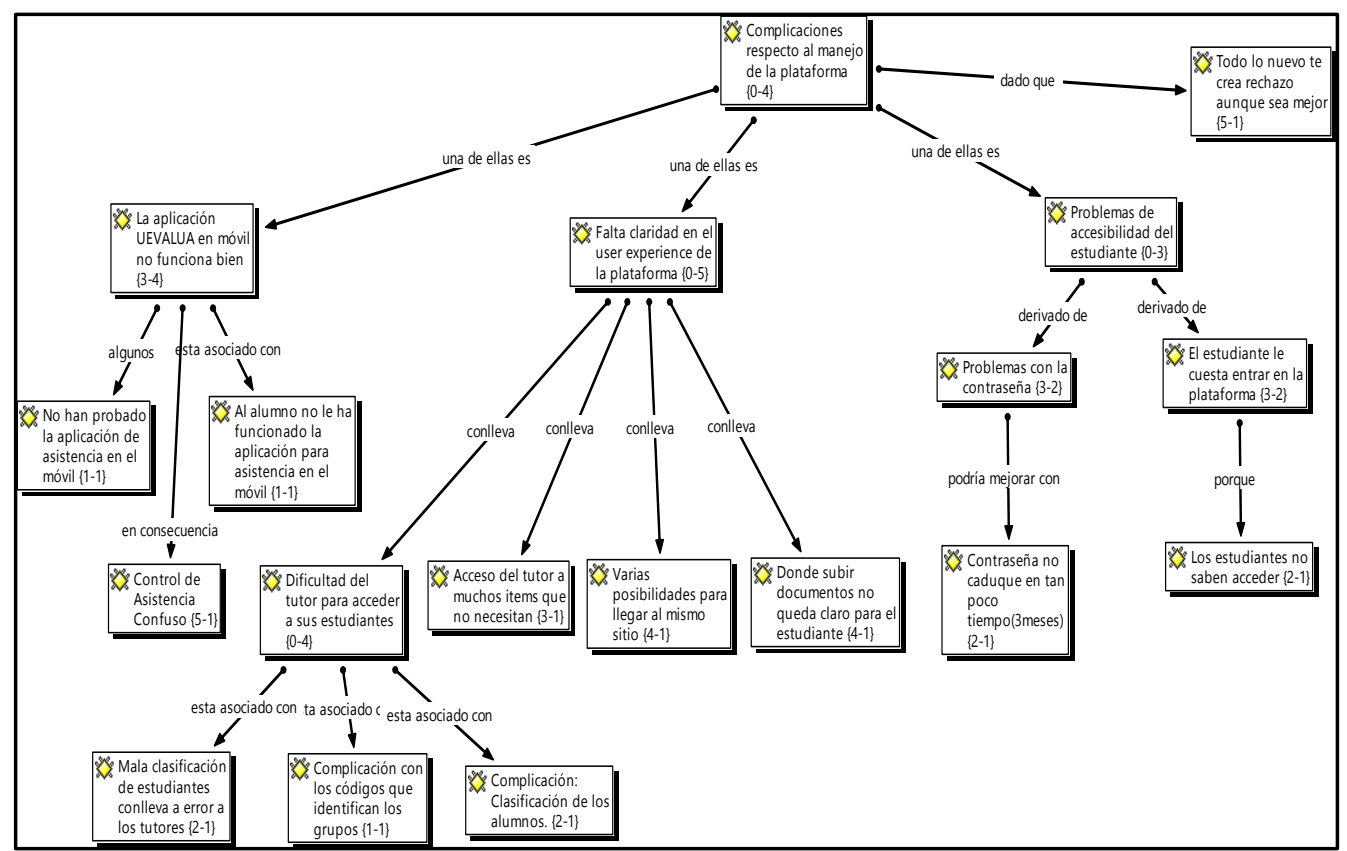

Fig 1. Árbol-estructura sobre las dificultades encontrados por los participantes durante la utilización de la nueva plataforma (Elaboración propia).

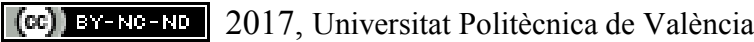


UEVALUA: El uso de las nuevas tecnologías para la evaluación y seguimiento de los estudiantes de Grado en Fisioterapia

En la subcategoría Aplicación móvil de la plataforma se destaca la dificultad que tienen algunos participantes para utilizar la aplicación móvil:

"Por ejemplo, cuando me pusieron la nota de las prácticas en el ordenador me salía y sin embargo, en el móvil no. Además con datos móviles no me funciona y con datos wifi si" (GD, A1, 26.52).

"Yo me la bajé pero la dejé de usar porque no funcionaba bien. Primero, que no me dejaba acceder y luego me dejó acceder pero no me aparecía nada de la información"(GD, A2, 27.13).

Otro motivo recogido por los participantes es que el control de asistencia es confuso. La aplicación móvil se pensaba que facilitaba el registro de asistencia del estudiante, sin embargo, las citas cuestionan la utilidad de la aplicación para la asistencia:

"La asistencia los tutores no la están haciendo por la plataforma, no conozco a ningún alumno que lo haga, lo hacen en papel, lo escanean y lo suben a la plataforma" (GD, TA1, 14.49).

"Como va con el pin, es un pin que tiene el tutor, tienes que estar detrás de ellos, yo hice el control de asistencia firmando, como se hacía antes" (GD, A3, 14.49).

"La idea era que vinieran y nosotros tikamos que han venido, pero no ha funcionado, no hemos podido hacerlo" (GD, TC3, 29.37).

Respecto a la Falta de claridad en el user experience de la plataforma los tutores académicos encontraron complicado el acceso a los estudiantes que estaban en prácticas en ese momento. Además recogieron que la plataforma permite llegar al mismo sitio mediante diferentes vías:

"El tutor en las rotaciones generales tiene acceso, puede puntuar y calificar el tutor clínico también a la rotación de neurología...Igual otra opción es que solo pueda acceder el tutor práctico de neurología a las calificaciones de neurología” (GD, A3, 15.25).

"Yo creo que el problema es localizar donde están las cosas, donde las ha subido el alumno, eso lleva mucho tiempo, te puedes tirar una hora intentando buscar el documento y tienes que escribir al alumno, no lo encuentro aqui donde lo has subido" (GD, TA2, 5.16).

"Puedes llegar al mismo sitio por muchas zonas, te pierdes" (GD, TC4, 02:00). Otro aspecto destacado dentro de la subcategoría Falta de claridad en el user experience de la plataforma es que los estudiantes tuvieron mucha dificultad para saber dónde subir los documentos de evaluación:

(c)) BY-NC-ND 2017, Universitat Politècnica de València Congreso IN-RED (2017) 
"A lo mejor te metes en la tarea de Anexo y no tiene un acceso directo a la tarea donde se sube el anexo...No sabes muy bien si lo estás subiendo bien o no" (GD, A1, 1.53).

“La plataforma en sí podía ser más intuitiva, podía ser más clara, porque te hace dudar donde subir las cosas y de donde sacar la información”" (GD, A3, 5.39).

En cuanto a la subcategoría Problemas de accesibilidad los participantes destacan la poca practicidad que tiene el hecho de que la contraseña se deba solicitar en persona en el campus. Además recogen que la contraseña caduca en 3 meses y deban volver al campus:

“El tema de la contraseña...me había caducado. Entonces he intentado volver a restaurarla y me dice que tengo que volver a la universidad. Entonces no puedo subir los documentos y la tutora está esperando"(GD, A4, 00.24).

Los tutores clínicos exponen que el estudiante no sabe acceder a la plataforma o tiene dificultad para hacerlo:

“ El chico que tenemos ahora es estupendo pero no ha sido capaz de subir nada a la plataforma... les cuesta mucho entrar en la plataforma” (GD, TC4, 21.24).

Por último, todos los participantes recogieron el hecho de que todo lo nuevo crea rechazo aunque sea mejor.Sin embargo, también señalaban que la plataforma aporta ventajas respecto al anterior sistema de evaluación, punto que se expone en el Epígrafe "Ventajas del uso de la nueva plataforma”. Esta reflexión fue realizada por todos los colectivos, los cuales reconocen el hecho de que cualquier sistema nuevo crea cierto grado de rechazo inicial puesto que les requiere mayor dedicación y tiempo:

"El tema de los tutores al fin y al cabo algo nuevo siempre da algo de miedo, es el tema de cómo funciona ...es costoso...pero creo que en un par de meses cuando ya se hayan familiarizado es algo fácil, sencillo, entre comillas solo cambiando algunas cosillas y rápido” (GD, $A 4,1.22)$.

"Ellos también ver una carga de trabajo añadida y no quieren hacer cambios, en los cambios siempre hay resistencia” (GD, TA3, 15.34).

"Lo que he vivido con mis compañeros al inicio es que esto es un lío. Pero es normal, a todos nos cuestan los cambios hasta que nos acostumbremos" (GD, TC4. 1:04).

\subsection{Formación inicial sobre el manejo de la plataforma}

Esta familia está compuesta por un total de 13 códigos. Se divide en dos subcategorías o subfamilias que muestran opiniones opuestas; mientras que para algunos la formación inicial ha sido clara y concisa, para otros ha sido insuficiente y no ha resuelto las dudas sobre el manejo de la plataforma y los documentos de evaluación. La figura que se muestra a continuación representa el árbol-estructura que muestra la relación entre los diferentes códigos sobre el manejo de la plataforma UEVALUA (Figura 2). 


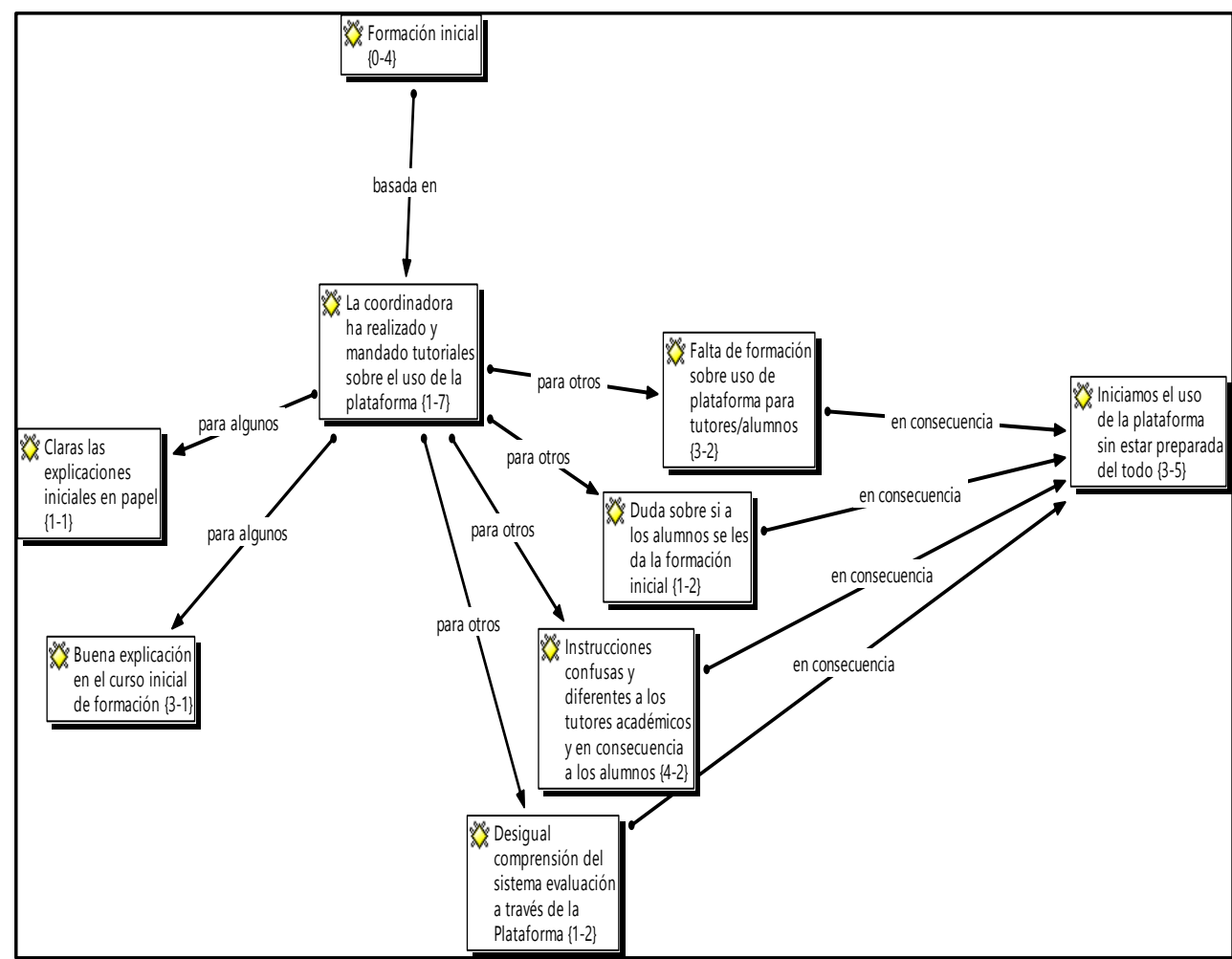

Fig 2. Árbol-estructura sobre la Formación inicial sobre el manejo de la plataforma UEVALUA (Elaboración propia).

Como aspecto más positivo de la formación se destaca que las explicaciones dadas han sido claras y por tanto, han tenido gran utilidad y han facilitado el manejo de la plataforma. Sin embargo, parte de los participantes enuncian la falta de formación sobre el uso de la plataforma:

“A mí como coordinadora, nadie me dio formación” ( GD, TC1, 27.18).

"Las explicaciones que nos mandaron en papel, me parecieron bastante claras" ( GD, TC4,02.04).

Los tutores académicos destacan la falta de unanimidad en las instrucciones dadas. Para ellos el principal motivo de esto es que se ha iniciado la plataforma sin estar preparados:

“Este año hemos vuelto a empezar a "matacaballo" por detrás del alumno” (GD, TA4, 30.40).

“Las instrucciones no son dadas iguales a todos los profesores" (GD, TA5, 2.35).

"El trimestre uno ha sido un poco locura porque no podían subir nada, no tenían el acceso, no han quitado la subida de diario reflexivo en la plataforma, sigue ahí, ha sido un poco complicado" (GD, TA3, 4.24).

(cc) EY-NC-ND 2017, Universitat Politècnica de València 


\subsection{Impacto en el rendimiento del estudiante}

Esta familia está compuesta por 30 códigos y se divide en 3 subcategorías o subfamilias: Formato papel vs Formato digital, la Actitud del estudiante actual y Feedback al estudiante. Esta familia emerge solamente de la experiencia de los tutores clínicos.

La figura que se muestra a continuación representa el árbol-estructura para ilustrar la relación entre los códigos de la familia de Impacto sobre el rendimiento del estudiante ( Figura 3).

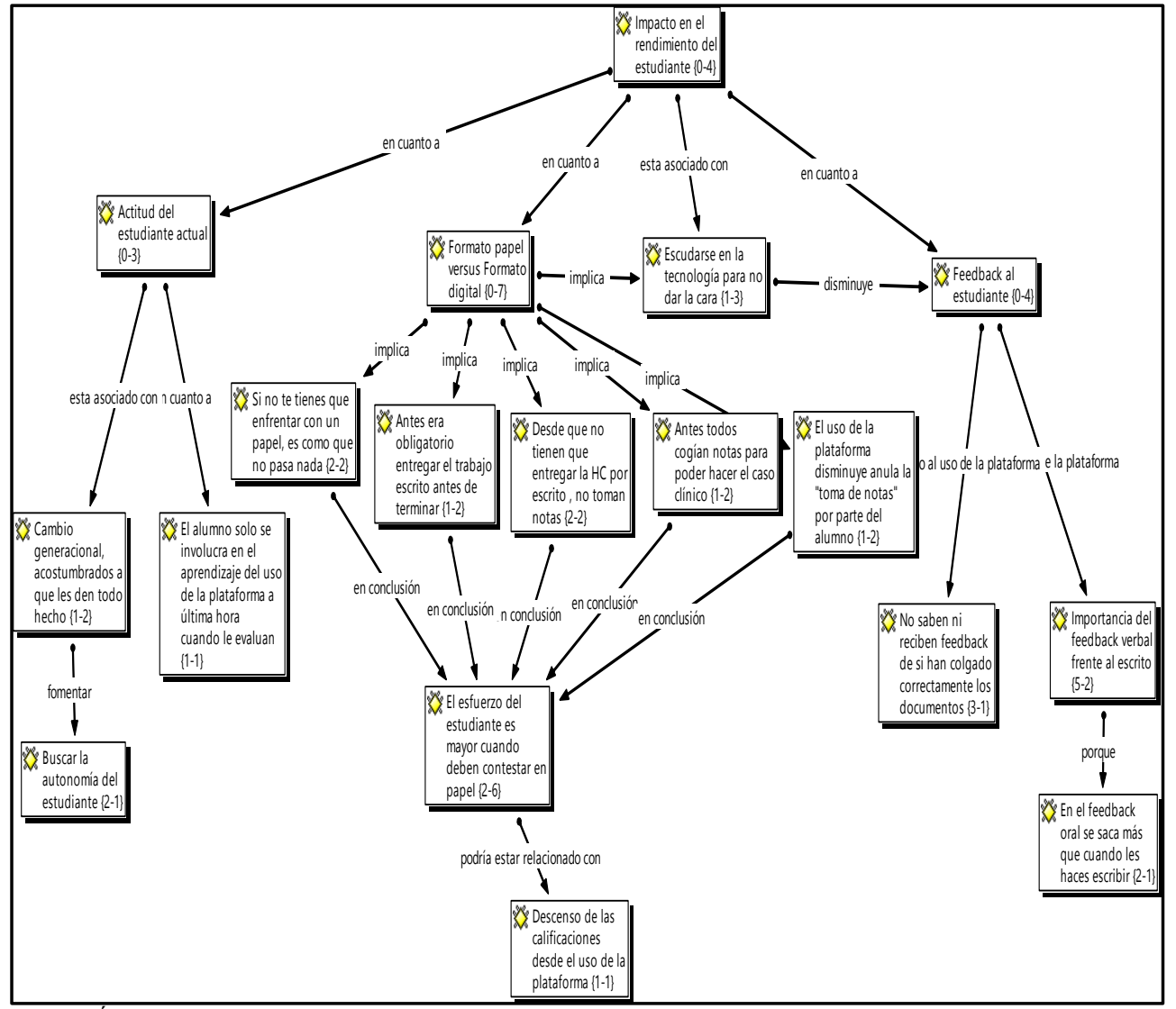

Fig 3. Árbol -estructura sobre el Impacto del uso de la plataforma UEVALUA en el rendimiento de los estudiantes.

En la subcategoría Formato papel vs Formato digital se destaca el hecho de la no obligatoriedad de entregar el trabajo por escrito al tutor clínico desde el uso de la plataforma. Este cambio parece haber tenido un impacto en la conducta de los estudiantes a la hora de tomar notas en el día a día de la práctica clínica:

"Antes, cuando se trabajaba en papel, era obligatorio entregar el trabajo en papel antes de que se finalizaran las prácticas" (GD, TC2, 34.39).

"He visto que desde que no tiene que traer la historia clínica en papel, no toman notas" (GD, TC3, 41.54).

(c)) EY-NC-ND 2017, Universitat Politècnica de València 
UEVALUA: El uso de las nuevas tecnologías para la evaluación y seguimiento de los estudiantes de Grado en Fisioterapia

El hecho de no tener que entregar nada por escrito al tutor evita al alumno la situación de tenerse que enfrentar cara a cara con la situación:

"Si no te enfrentas a alguien con un papel en ese mismo momento, es como si no pasa nada" ( GD, TC3, 33.27).

"El papel es una manera de enfrentarse cara a cara. Con el ordenador está la pantalla delante, pero no la persona que te lo va a corregir" (GD, TC1, 34.21).

$\mathrm{Y}$ esta distancia parece tener un impacto en el esfuerzo del estudiante y en las calificaciones obtenidas en la práctica clínica:

"A mí me parece que se esforzaban más cuando tenían que entregarlo por escrito, contestar a ese escrito" ( GD, TC3, 18.08).

"Las notas eran antes mucho más altas que ahora" (GD, TC2, 11.03).

En la subcategoría Feedback al estudiante vuelve a emerger el tema del papel, pues cuando el alumno debía entregarlo en papel, el tutor se sentaba con él para darle un feedback oral:

"Parece que se saca más de ellos cuando les haces escribir" (GD, TC2, 0.23).

"Tengo una conversación con el alumno, que es mucho mejor que dejarlo por escrito, le aporta mucho más" (GD, TC1, 0.17).

Por otro lado, se percibe una falta de feedback respecto a la confirmación de si han subido a la plataforma los documentos correctamente:

"No sé si hice bien todo, si todos pueden ver lo que yo he colgado" (GD, A5, 3.07).

"Hice la clínica al principio del trimestre pasado y no tengo manera de saber si lo hecho bien, si lo hecho mal..."(GD, A3, 3.50).

Para los participantes el uso de la plataforma digital disminuye las opciones de dar un feedback verbal al estudiante. Esta situación permite al estudiante protegerse tras la pantalla de su ordenador, "Escudarse en la tecnología para no dar la cara":

"La gente se escuda mucho en la tecnología para no dar la cara. Tú lo puedes leer en el ordenador, sin que nadie vea lo que estás leyendo, Aunque la coordinadora de la universidad esté viendo su trabajo, tú no te enfrentas ni a esa persona ni a ese momento" ( GD, TC3, 32.34).

En la subcategoría Actitud del estudiante actual se destaca la percepción de los participantes respecto al cambio generacional que observan en los estudiantes:

"Han cambiado mucho las generaciones...Yo tampoco soy muy vieja pero ha habido un cambio brutal, están acostumbrados a que todo se lo dan hecho, y si de les exige, encima la culpa es tuya" ( GD, TC1, 43.07).

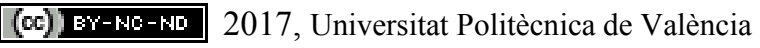
Congreso IN-RED (2017) 
“Es una pena porque para nosotros las prácticas era lo mejor de la carrera” ( $G D, T C 3,43.28)$.

Otro aspecto destacado por los participantes en esta subcategoría de Actitud del estudiante actual es la necesidad de buscar la Autonomía del estudiante en su trabajo diario en las prácticas clínicas:

"Yo les hecho una mano pero intento que sean ellos los que saquen el contenido de ese paciente" GD; TC4; 36.54).

"Yo les hago que ellos trabajen en casa, sin tenerles que obligar ni perseguirles. Si les digo lo que tienen que hacer les estoy cerrando la cabeza” ( GD, TC1, 39.54).

\subsection{Ventajas del uso de la plataforma}

Esta familia está compuesta por 15 códigos y se divide en 3 subcategorías o subfamilias: Sencilla porque no necesita conocimientos de informática para usarla, La plataforma es útil y La plataforma mejora la comunicación estudiante vs Cínica vs tutor académico. La figura representa el árbol-estructura que ilustrar los códigos de esta familia ( Figura 4).

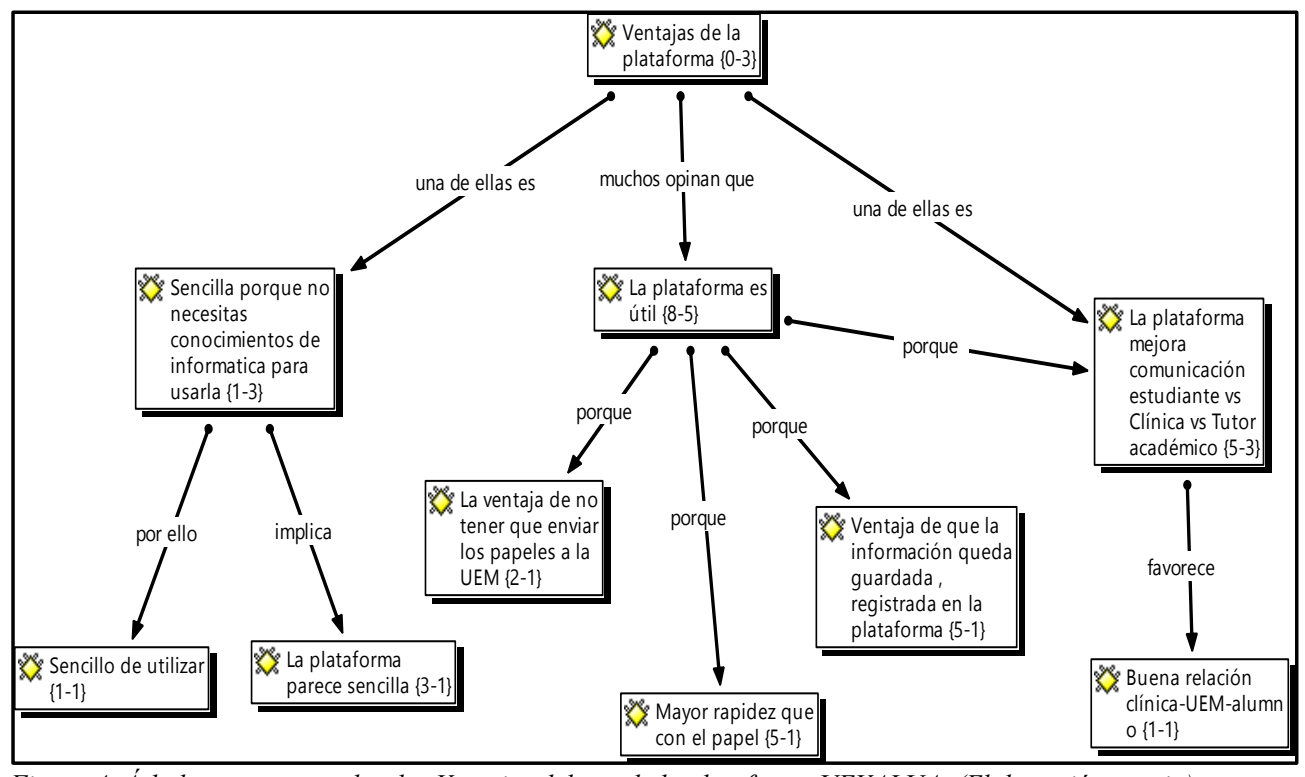

Figura 4. Árbol-estructura sobre las Ventajas del uso de la plataforma UEVALUA. (Elaboración propia).

En la subcategoría Sencilla porque no necesita conocimientos de informática para usarla se destaca la ventaja de la sencillez del uso de la plataforma:

"Es muy intuitiva, no necesitas conocimientos informáticos ni nada” (GD, TC2, 26.39).

“La plataforma es muy sencilla a priori, es muy visual” ( GD, TC3, 25.49).

"Yo creo que en un par de meses cuando ya se haya familiarizado, es algo fácil, sencillo y rápido" (GD, A4, 1.30).

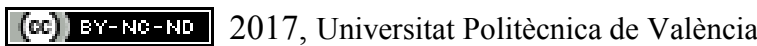
Congreso IN-RED (2017) 
UEVALUA: El uso de las nuevas tecnologías para la evaluación y seguimiento de los estudiantes de Grado en Fisioterapia

En la subfamilia de la Plataforma es útil se destaca la mayor rapidez en el trámite de los documentos con la universidad. Al mismo tiempo, el hecho de que los documentos queden registrados en la plataforma es una ventaja compartida por todos los participantes:

"Es una herramienta que me resulta útil y cómoda y evita tener papeles para arriba y para abajo" ( GD, TC2, 49.48).

"Todo está ahí cargado. Si una compañera se da la baja, yo puedo entrar con su perfil. No tienes que mandar nada por correo" ( GD, TC4, 44.59)

La última subcategoría de esta familia es La plataforma mejora la comunicación estudiante vs Cínica vs tutor académico:

"Reune tanto a la universidad, como a nosotros como a las prácticas, entonces es un elemento bastante útil” (GD, A3, 9.34)

"La mejora importante de esa plataforma es que los dos tutores, clínico y académico estan juntos, entonces el alumno recibe un feedback en la misma plataforma de los dos "(GD, TA2, 14.09)

\subsection{Propuestas de mejora}

Esta familia está compuesta por 24 códigos y se divide en 3 subcategorías o subfamilias, cada una perteneciente a uno de los tres colectivos que han formado parte del estudio: Propuestas de los tutores clínicos, Propuestas de los estudiantes y Propuestas de los tutores académicos. La figura representa el árbol-estructura para ilustrar la relación entre los diferentes códigos que componen esta familia ( Figura 5).

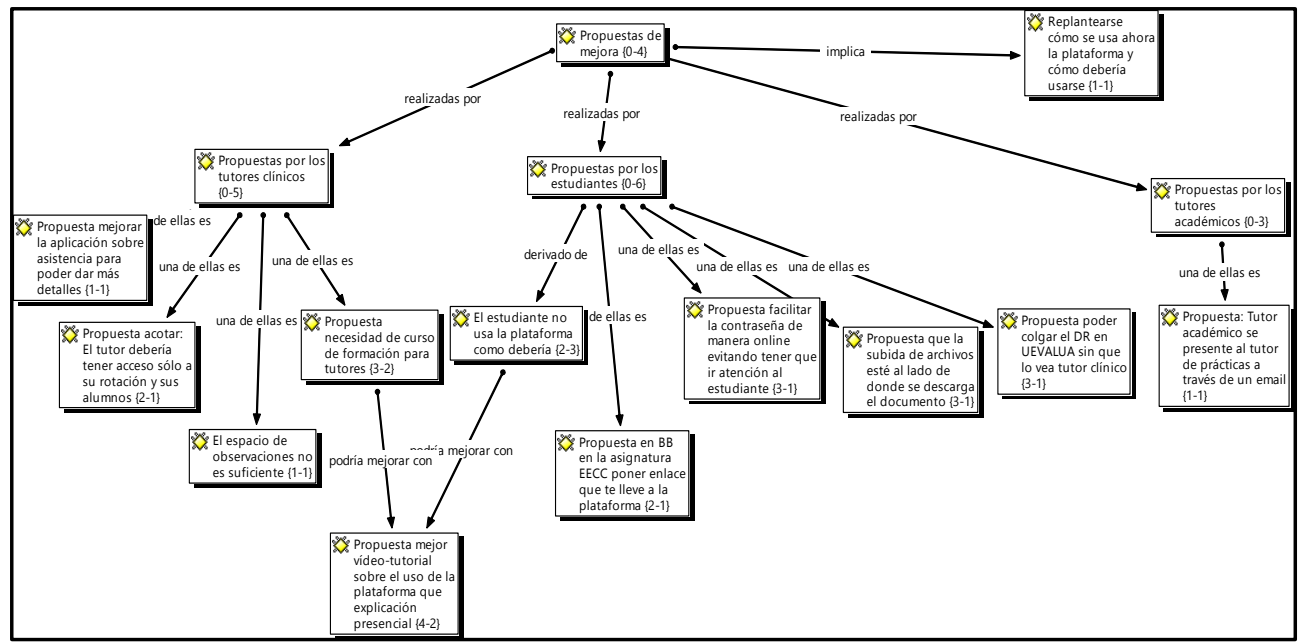

Fig 5. Árbol-estructura sobre las Propuestas de mejora de la plataforma UEVALUA.

La subcategoría Propuestas de los tutores clínicos se centra en mejorar la aplicación del control de asistencia para poder dar más detalles sobre el desempeño del alumno y en limitar el acceso de cada tutor al grupo de alumnos que está tutorizando:

“Hay que acotarlo. Que te salgan sólo tus alumnos” ( GD, TC4,27.52)

(c)) BY-NC-ND 2017, Universitat Politècnica de València 
"Echo de menos, poder poner "Fulanito" ha venido hoy pero ha llegado tarde y debe recuperar 2 horas. En observaciones no es suficiente." ( GD, TC3, 30.01).

La subcategoría de Propuestas de los estudiantes recoge sus sugerencias para facilitar el uso de la plataforma. Los estudiantes opinan que se debería simplificar el proceso para la obtención de la contraseña:

"Podrían facilitar la contraseña a través de la página web y evitar la espera en atención al estudiante" (GD, A2, 5.23)

Otra propuesta de mejora es que el enlace a la nueva plataforma se vinculase al espacio del Blackboard que tienen las materias de Estancias Clínicas y Practicum, pudiendo acceder directamente desde allí a UEVALUA:

"Otra cosa que estaría bien sería en la asignatura del campus virtual poner un enlace que te llevara a la plataforma" (GD, A2, 19.51)

Las propuestas de mejora de los tutores académicos se recogen en la subcategoría Propuestas del tutor académico. Como acciones de mejora proponen que el tutor académico y el tutor clínico se pongan en contacto a través de la plataforma antes de la rotación del estudiante:

"Estaría bien mandar un mensaje a los tutores "yo soy tutora académica de este alumno, ante cualquier cosa póngase en contacto conmigo"”,(GD, TA1, 20.17)

En relación con esta familia de Propuestas de mejora, una acción a tener en cuenta sería la de elaborar un vídeo- tutorial para explicar el uso de la plataforma. Los participantes se plantean que cómo usan la plataforma en la actualidad no tiene porqué ser la mejor forma de hacerlo:

"Hay que plantearse, cómo estamos usando hoy la plataforma, y cómo la podríamos utilizar” ( GD, TC2, 46.47)

"Un vídeo explicativo de 1 minuto.. Un tutorial que sea corto, conciso, (GD, A3, 16.42)

\section{CONCLUSIONES}

La experiencia de los participantes respecto al uso de la plataforma refleja el concepto de que todo lo nuevo crea un rechazo inicial. En su vivencia, los participantes han encontrado complicaciones en cuanto al manejo de la plataforma y ventajas en el uso de la misma. Todos los participantes destacan como principal complicación una deficiente User Experience, que implica una falta de claridad en la navegación por la plataforma, que, a priori, describen como fácil de utilizar.

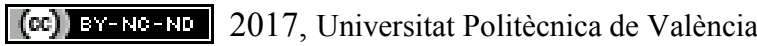
Congreso IN-RED (2017) 
Respecto al impacto de la herramienta en la comunicación entre estudiantes y tutores, los resultados recogen un impacto negativo en el feedback estudiante-tutor clínico al afirmar, el tutor, que el estudiante se escuda en la tecnología para no dar la cara, lo que disminuye la comunicación oral entre ellos.

En cuanto al impacto de la herramienta en el proceso de evaluación, han sido los tutores clínicos, los que han destacado que el uso de la plataforma ha repercutido negativamente en el rendimiento del estudiante, al considerar que el nivel de responsabilidad de este último se ve afectado al no tener que entregar ningún documento en papel.

Respecto a las ventajas destacadas por todos grupos, se destaca el hecho de que el uso de la plataforma supone un espacio de registro para todos los documentos de evaluación y seguimiento, lo que agiliza además los trámites de calificación entre la Universidad y el centro de prácticas externo. El hecho de que exista un espacio común de comunicación entre estudiante-tutor clínico-tutor académico, es también considerado una ventaja por todos ellos.

Las propuestas de mejora son un elemento común en todos los grupos de discusión y apuestan por simplificar la navegación del usuario en la plataforma, reforzar la formación inicial a todos los grupos buscando la homogeneidad en la misma y mejorar el funcionamiento de la aplicación móvil.

Podemos concluir que la novedad de esta plataforma, implementada hace sólo 3 meses, podría conllevar el hecho de que sean las complicaciones el tema más destacado del estudio. Consideramos que la continuidad en el uso de la plataforma por parte de todos los actores participantes, disminuirá parte de las mismas ya que el nivel de manejo será mayor y los usuarios se podrán beneficiar de todo el potencial de la misma. Las acciones de mejora propuestas podrán ayudarnos a optimizar el uso de esta nueva plataforma de evaluación de prácticas clínicas externas.

\section{Referencias}

Blández, J. (2000). La investigación-acción, un reto para el profesorado. Barcelona: Inde Publicaciones.

Centre for Reviews and Dissemination. Incorporating Qualitative Evidence in or alongside effectivenesss reviews. In Centre for Reviews and Dissemination (eds). Systematic Reviews: CDR's guidance for undertaking reviewa in health care. USA: Centre for Reviews and Dissemination. University of York; 2008.

Fernández-Pampillón Cesteros, A. (2009). Las plataformas e-learning para la enseñanza y el aprendizaje universitario en Internet.

(c)) EY-NC-ND 2017, Universitat Politècnica de València Congreso IN-RED (2017) 
Giorgi, A. (2005). The phenomenological movement and research in the human sciences. Nursing science quarterly, 18(1), 75-82.

Giorgi, A. (2012). The descriptive phenomenological psychological method. Journal of Phenomenological psychology, 43(1), 3-12.

Gutiérrez Cárdenas, L., \& Leco Berrocal, M. I. (2015). Del "Cuaderno de Prácticas" a la "Plataforma de Evaluación de Prácticas Clínicas "en odontología.

Klopper, H. (2008). The qualitative research proposal. Curationis, 31(4), 62-72.

Ley 44/2003 de 21 de noviembre para la ordenación de profesionales sanitarios, num. 280 de 22 de noviembre de 2003.

Lincoln, Y. S., y Guba, E. G. (1985). Naturalist inquiry. California: Sage.

Muñoz, J. (2008). Análisis cualitativo de datos textuales con ATLAS. ti 5. Barcelona: Universitat Autónoma de Barcelona, 2005.

Orden CIN 2135/2008 de 3 de julio, por la que se establecen los requisitos para la verificación de los títulos universitarios oficiales que habiliten para el ejercicio de la profesión de Fisioterapeuta, num 1743 de julio de 2008.

Pérez Serrano, G. (1994). Investigación cualitativa. Retos e interrogantes. Madrid: La Muralla.

Real Decreto 592/2014 de 11 de julio por el que se regulan las prácticas académicas externas de los estudiantes universitarios, num. 184, de 30 de julio de 2014.

Reynés, M. R. M., Bertos, J. E., Vila, M. C., \& Ramón, M. I. (2014). Seguimiento, tutorización y evaluación de prácticas externas mediante plataforma virtual de trabajo colaborativo: la experiencia en el Grado de Geografía. Revista de investigación en educación, 2(12), 254-267.

Sheldon, T. A. (2005). Making evidence synthesis more useful for management and policymaking.

Starks, H., \& Trinidad, S. B. (2007). Choose your method: A comparison of phenomenology, discourse analysis, and grounded theory. Qualitative health research, 17(10), 1372-1380.

Vélez Vélez, E., González Hernández, A., Hernández Salinero, F., Rodríguez, P., \& Santiago, M. (2012). Seguimiento y evaluación de las prácticas clínicas tuteladas con ayuda de la plataforma Moodle: Experiencia piloto y propuesta de mejora. Enfermería Global, 11(26), 86-101.

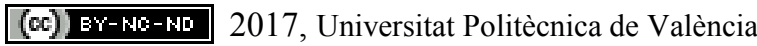

